\title{
AN INTERNAL CHARACTERIZATION OF INESSENTIAL OPERATORS
}

\author{
PIETRO AIENA
}

(Communicated by John B. Conway)

\begin{abstract}
We characterize the ideal of inessential operators $I(E)$ on a complex Banach space $E$ as the largest ideal of the class $A(E)$ of all bounded linear operators $A$ having the property that the restrictions $A \mid M$ of $A$ on any closed infinite-dimensional invariant subspace $M$ may be.
\end{abstract}

There are several characterizations of the closed ideal of inessential operators $I(E)$ on a complex Banach space $E$. The first one, used by D. C. Kleinecke when he introduced this class [3], is the following: $I(E)=$ the inverse canonical image of the radical of the Calkin algebra $B(E) / K(E)$, where $B(E)$ and $K(E)$ denote the Banach algebra of all bounded linear operators and the closed ideal of all compact linear operators acting on $E$, respectively. It is well known that for all the operators of $I(E)$ the basic statements of the Riesz theory hold (cf. [1 or 2]). Moreover if we denote by

$$
R(E)=\{A \in B(E): \lambda I-A \text { is a Fredholm operator for each complex } \lambda \neq 0\}
$$

the class of all Riesz operators, we have (cf. [2, Proposition 52.6])

$$
I(E)=\text { the uniquely determined largest ideal of } R(E) \text {. }
$$

Denote by $\Phi(E)$ the set of all Fredholm operators on $E$ and by $n(A)$ the dimension of the kernel $N(A)$ for any $A \in B(E)$. In [5] it is shown that

$$
I(E)=\{A \in B(E): n(S-A)<\infty \text { for each } S \in \Phi(E)\} .
$$

The purpose of this note is to give an internal characterization of inessential operators by observing their behavior on a certain class of subspaces of $E$. We shall prove that $I(E)$ is the largest ideal of the class $A(E)$ of all bounded linear operators $A$ having the property that the restrictions of $A$ on any closed infinite-dimensional invariant subspace $M$ for $A$ is not bijective. In the sequel we shall use the terminology of Heuser's book [2].

The following characterization of the inessential operators is due to A. Pietsch [4]. We shall give a very simple proof starting from Schechter's characterization $(*)$.

THEOREM 1. $I(E)=\{A \in B(E): n(I-B A)<\infty$ for each $B \in B(E)\}$.

Proof. Let $A \in I(E)$. Since $I(E)$ is an ideal, the operator $B A$ belongs to $I(E)$ and taking $S=I$ in (*), the identity on $E$, we have $n(I-B A)<\infty$ for each $B \in B(E)$.

Received by the editors December 4, 1986.

1980 Mathematics Subject Classification (1985 Revision). Primary 47B05.

Key words and phrases. Inessential and Riesz operators.

This work was supported in part by the Italian Research Science Foundation (C.N.R.). 
Conversely let $A \notin I(E)$. Then there exists a Fredholm operator $S$ such that $(* *)$

$$
S x=A x \quad \text { for each } x \in M=N(S-A),
$$

where $M$ is infinite dimensional. Since $S \in \Phi(E)$, by Atkinson characterization, there exists an operator $U \in B(E)$ and a finite-dimensional operator $K \in B(E)$ such that $U S=I-K$ (see [2, Proposition 24.1]). It follows from the equality (**) that $U S x=U A x=(I-K) x$ for each $x \in M$, thus $(I-U A) x=K x$ for each $x \in M$. If $(I-U A) \mid M$ denotes the restriction of $I-U A$ to the subspace $M$, we have $n(I-U A) \geq n[(I-U A) \mid M]=n[(K \mid M)]=\infty$ so $A$ does not belong to the set $\{A \in B(E): n(I-B A)<\infty$ for each $B \in B(E)\}$.

The following result will be useful in the proof of the subsequent main result.

THEOREM 2. If $A$ is a Riesz operator, then $A \in A(E)$.

ProOF. Let us suppose $A \in R(E)$ and let $M$ be a closed invariant subspace for $A$ such that the restriction $A \mid M$ of $A$ on $M$ has a inverse $(A \mid M)^{-1}$. Since $E$ is a complex Banach space, $A \mid M$ is still a Riesz operator (see [2, Proposition 52.7]). Moreover the operators $A \mid M$ and $(A \mid M)^{-1}$ commute so their product $I \mid M$ is a Riesz operator (cf. [2, Proposition 52.3]), hence $M$ is finite dimensional.

Now we can give our internal characterization.

THEOREM 3. $I(E)$ is the uniquely determined maximal ideal of $\mathcal{A}(E)$-operators. Each ideal of $A(E)$-operators is contained in $I(E)$.

PrOOF. Let $G$ be any ideal of $A(E)$-operators (such ideals do exist, e.g. the ideal $K(E)$ ). Furthermore, let $A$ be a fixed element of $G$ and $B$ any bounded operator on $E$. Then $B A \in G, N(I-B A)$ is a closed subspace invariant under $B A$, moreover the restriction of $B A$ to $N(I-B A)$ coincides with the restriction of the identity to $N(I-B A)$ and has therefore a bounded inverse. It follows from the definition of $A(E)$-operators that $n(I-B A)$ must be finite. Pietsch's characterization now implies $A \in I(E)$. Hence any ideal of $A(E)$-operators is contained in $I(E)$. On the other hand, the ideal $I(E)$ is itself an ideal of Riesz operators, and therefore of $A(E)$-operators (see Theorem 2). It follows that $I(E)$ is the uniquely determined maximal ideal of $A(E)$-operators.

At this point I wish to thank H. Heuser for several valuable discussions on the topic of this paper. The author is also indebted to the referee for useful suggestions and especially for the simple and short proof of Theorem 2.

\section{REFERENCES}

1. S. Caradus, W. E. Pfaffenberger and B. Yood, Calkin algebras and algebras of operators in Banach spaces, Dekker, New York, 1974.

2. H. Heuser, Functional analysis, Wiley, New York, 1982.

3. D. Kleinecke, Almost finite, compact and inessential operators, Proc. Amer. Math. Soc. 14 (1963), 863-868.

4. A. Pietsch, Inessential operators in Banach spaces, Integral Equations Operator Theory 1 (1978), 588-591.

5. M. Schechter, Riesz operators and Fredholm perturbations, Bull. Amer. Math. Soc. 74 (1968), 1139-1144.

Dipartimento di Matematica ed Applicazioni, Università di Palermo, 90123 Via ArChirafi 34, Palermo, italy 